\section{CARDIOPROTECTION}

\section{Stratifying the effects of SGLT2i}

Two prespecified analyses of the DECLARETIMI 58 trial, presented at ACC.19, show that in patients with type 2 diabetes mellitus (T2DM), the sodium-glucose cotransporter 2 inhibitor (SGLT2i) dapagliflozin has greater cardiovascular benefit in those with heart failure with reduced ejection fraction (HFrEF) at baseline and in those with prior myocardial infarction (MI).

Of the 17,160 patients with T2DM in the trial, $3.9 \%$ had HFrEF (ejection fraction (EF) $<45 \%$ ), $7.7 \%$ had heart failure (HF) without known reduced $\mathrm{EF}$ and $88.4 \%$ had no history of HF. Compared with placebo, dapagliflozin reduced the risk of cardiovascular death and hospitalization for $\mathrm{HF}$ to a greater extent in patients with $\mathrm{HFrEF}$ (HR 0.62, 95\% Cl 0.45-0.86) than in patients without HFrEF (HR 0.88, 95\% $\mathrm{Cl} 0.76-1.02, P=0.046$ for interaction). This difference was driven by a strong reduction in cardiovascular death and all-cause death in patients with HFrEF but not in those without HFrEF. Among patients without HFrEF, the effect of dapagliflozin was similar in patients with $\mathrm{HF}$ without known reduced $\mathrm{EF}$ and in patients without a history of HF.

Patients with prior MI (20.9\%) treated with dapagliflozin had a $16 \%$ reduction in the relative risk and a $2.6 \%$ reduction in the absolute risk of major adverse cardiovascular events (MACE) compared with the placebo group (15.2\% versus $17.8 \%$; HR $0.84,95 \%$ $\mathrm{Cl} 0.72-0.99, P=0.039$ ), mainly driven by reductions in the rate of recurrent $\mathrm{MI}$. By contrast, dapagliflozin had no effect on the risk of MACE in patients without a history of $\mathrm{MI}(7.1 \%$ versus $7.1 \%)$. The relative risk reduction in cardiovascular death and hospitalization for HF with dapagliflozin was similar in patients with or without prior MI, but the absolute risk reduction tended to be greater in patients with prior MI. "Whether this benefit could be expanded to the acute phase of Ml or to patients with prior Ml even if they do not have T2DM should be addressed in future studies," note the researchers. Irene Fernández-Ruiz

ORIGINAL ARTICLES Kato, E. T. et al. Effect of dapagliflozin on heart failure and mortality in type 2 diabetes mellitus. Circulation https://doi.org/10.1161/CIRCULATIONAHA.119.040130 (2019) | Furtado, R. H. M. et al. Dapagliflozin and cardiovascular outcomes in patients with type 2 diabetes and prior myocardial infarction: a sub-analysis from DECLARE TIMI-58 trial. Circulation https://doi.org/10.1161/CIRCULATIONAHA.119.039996 (2019) FURTHER READING Nassif, M. \& Kosiborod, M. Effect of glucose-lowering therapies on heart failure. Nat. Rev. Cardiol. 15, 282-291 (2018)

\title{
Immediate angiography does not improve outcomes after OHCA without STEMI
}

A strategy of immediate coronary angiography and percutaneous coronary intervention (PCI) does not improve outcomes following successful resuscitation after out-of-hospital cardiac arrest (OHCA) in the absence of ST-segment elevation myocardial infarction (STEMI). This finding from the COACT study was presented at ACC.19.

Recommended care after successful cardiac resuscitation includes targeted temperature management, vital-organ support and treatment of the underlying cause of the cardiac arrest; however, the underlying cause is not always immediately known, leading to uncertainty about the appropriate treatment strategy. The COACT investigators hypothesized that immediate angiography might reduce the time delay to PCI in indicated patients and thereby improve myocardial salvage.

In this multicentre trial, 552 patients with cardiac arrest but no signs of STEMI were randomly assigned to undergo immediate coronary angiography or coronary angiography that was delayed until after neurological recovery. All patients underwent PCI if indicated.

After 90 days of follow-up, survival was $64.5 \%$ in the immediate-angiography group compared with $67.2 \%$ in the delayed-angiography group (OR 0.89, 95\% CI $0.62-1.27, P=0.51$ ). Of note, the median time to achieving target temperature was $5.4 \mathrm{~h}$ and $4.7 \mathrm{~h}$ in each group, respectively. "A later achievement of target temperature might have attenuated any potential benefit gained from immediate coronary angiography," speculate the investigators. Another factor influencing the results might be the proportion of patients with stable coronary artery lesions versus thrombotic occlusions (only $5 \%$ in the COACT trial).

Gregory B. Lim

ORIGINAL ARTICLE Lemkes, J. S. et al. Coronary angiography after cardiac arrest without ST-segment elevation. N. Engl.J. Med. https://doi.org/10.1056/NEJMoa1816897 (2019) FURTHER READING Berg, D. D. et al. Key components of a community response to out-of-hospital cardiac arrest. Nat. Rev. Cardiol. https://doi.org/10.1038/s41569-019-0175-4 (2019)

\section{ATRIAL FIBRILLATION}

\section{Delaying cardioversion for recent-onset $\mathrm{AF}$}

Patients presenting to the emergency department with recent-onset atrial fibrillation (AF) do not benefit more from early cardioversion compared with a wait-and-see approach in achieving a return to sinus rhythm at 4 weeks. The results of the RACE 7 ACWAS multicentre, randomized, noninferiority trial were presented at the European Heart Rhythm Association Congress 2019, and simultaneously published in the NEJM.

Patients presenting to the hospital with symptomatic and recent-onset $(<36 \mathrm{~h}) \mathrm{AF}$ were randomly assigned to be managed by a wait-and-see approach $(n=218$; delayedcardioversion group) or to the standard care of early cardioversion ( $n=219$; early-cardioversion group). The wait-and-see approach involved the administration of rate-control medication for symptom relief; if AF was still present $48 \mathrm{~h}$ after symptom onset, patients were referred for cardioversion.

At the 4-week follow-up, $91 \%$ of patients in the delayed-cardioversion group and $94 \%$ of patients in the early-cardioversion group were in sinus rhythm (between-group difference -2.9 percentage points, $95 \% \mathrm{Cl}$ -8.2 to $2.2, P=0.005$ for noninferiority). In the early-cardioversion group, $16 \%$ of patients experienced a spontaneous return to sinus rhythm before initiation of cardioversion, whereas sinus rhythm was achieved in $78 \%$ of patients who underwent cardioversion. In the delayed-cardioversion group, $69 \%$ of patients experienced a spontaneous return to sinus rhythm within $48 \mathrm{~h}$, compared with $28 \%$ of patients who required cardioversion.

"The approaches to treating patients with recent-onset [AF] in the emergency department vary greatly," remark the investigators. The RACE 7 ACWAS findings suggest that many patients with stable, symptomatic AF do not necessarily need to undergo immediate cardioversion, thus reducing the risk of potential complications.

Karina Huynh

ORIGINAL ARTICLE Pluymaekers, N. A. H. A. et al. Early or delayed cardioversion in recent-onset atrial fibrillation. N. Engl.J. Med. https://doi.org/10.1056/NEJMoa1900353 (2019) FURTHER READING Dobrev, D. et al. Postoperative atrial fibrillation: mechanisms, manifestations and management. Nat. Rev. Cardiol. https://doi.org/10.1038/s41569-019-0166-5 (2019) 